\title{
BMJ Open General practitioners' attitudes towards the management of dental conditions and use of antibiotics in these consultations: a qualitative study
}

\author{
Anwen L Cope, ${ }^{1}$ Fiona Wood, ${ }^{2}$ Nick A Francis, ${ }^{2}$ Ivor G Chestnutt ${ }^{1}$
}

To cite: Cope AL, Wood F, Francis NA, et al. General practitioners' attitudes towards the management of dental conditions and use of antibiotics in these consultations: a qualitative study. BMJ Open 2015;5: e008551. doi:10.1136/ bmjopen-2015-008551

- Prepublication history for this paper is available online. To view these files please visit the journal online (http://dx.doi.org/10.1136/ bmjopen-2015-008551).

Received 20 April 2015 Revised 28 July 2015 Accepted 7 August 2015

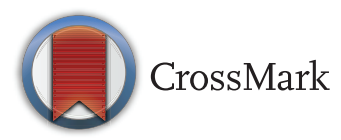

${ }^{1}$ Applied Clinical Research and Public Health, School of Dentistry, Cardiff University, Cardiff, UK

${ }^{2}$ Cochrane Institute of

Primary Care and Public Health, School of Medicine, Cardiff University, Cardiff, UK

Correspondence to Dr Anwen L Cope; copea1@cardiff.ac.uk

\section{ABSTRACT}

Objectives: This study aimed to produce an account of the attitudes of general practitioners (GPs) towards the management of dental conditions in general practice, and sought to explore how GPs use antibiotics in the treatment of dental problems.

Design: Qualitative study employing semistructured telephone interviews and thematic analysis.

Participants: 17 purposively sampled GPs working in Wales, of which 9 were male. The median number of years since graduation was 21. Maximum variation sampling techniques were used to ensure participants represented different Rural-Urban localities, worked in communities with varying levels of deprivation, and had differing lengths of practising career.

Results: Most GPs reported regularly managing dental problems, with more socioeconomically deprived patients being particularly prone to consult. Participants recognised that dental problems are not optimally managed in general practice, but had sympathy with patients experiencing dental pain who reported difficulty obtaining an emergency dental consultation. Many GPs considered antibiotics an acceptable first-line treatment for acute dental problems and reported that patients often attended expecting to receive antibiotics. GPs who reported that their usual practice was to prescribe antibiotics were more likely to prioritise patients' immediate needs, whereas clinicians who reported rarely prescribing often did so to encourage patients to consult a dental professional.

Conclusions: The presentation of patients with dental problems presents challenges to GPs who report concerns about their ability to manage such conditions. Despite this, many reported frequently prescribing antibiotics for patients with dental conditions. This may contribute to both patient morbidity and the emergence of antimicrobial resistance. This research has identified the need for quantitative data on general practice consultations for dental problems and qualitative research exploring patient perspectives on reasons for consulting. The findings of these studies will inform the design of an intervention to support patients in accessing appropriate care when experiencing dental problems.

\section{Strengths and limitations of this study}

- This paper presents a detailed analysis of the attitudes of medical practitioners towards the management of dental problems in general practice and their use of antibiotics in such consultations. It therefore provides a novel perspective on an issue that has received relatively little research attention to date.

- The use of qualitative methods facilitated an in-depth exploration of practitioners' perceptions of dental consultations and their reasons for prescribing antibiotics during these appointments.

- Maximum variation sampling techniques allowed researchers to obtain the views of a wide range of practitioners. However, findings may not be generalisable to all general practitioners (GPs) and it is possible that GPs that did not want to take part would have provided other insights on the topic.

- In this study GPs identified reasons why patients may consult with dental problems. However, more patient-centred research is indicated to investigate the underlying motivations for consulting.

\section{INTRODUCTION}

Clinical guidelines recommend that surgical intervention by a dental practitioner should be the first-line treatment for acute dental conditions. ${ }^{1}$ Despite this there is evidence that some patients will seek care from their general practitioner (GP). In a retrospective study of the General Practice Morbidity Database for Wales, approximately $0.3 \%$ of attendances at medical practices in Wales in 1996 were for oral or dental problems, equating to approximately 6.9 consultations per 1000 patient-years for tooth-related problems. $^{2}$ In comparison, similar studies of general practice have reported 13 consultations per 1000 patient-years for acute otitis media and 5 consultations per 1000 patient- 
years for laryngitis. ${ }^{3}{ }^{4}$ Dental problems may therefore represent a similar burden on general practice as some other common conditions of the head and neck.

Patients who consult their GP due to tooth-related problems are unlikely to receive a surgical intervention and have a greater likelihood of being prescribed a systemic antibiotic compared to individuals who consult a dentist with similar symptoms. ${ }^{5}$ However, antibiotic therapy alone is largely ineffective for acute dental problems related to the dental pulp, as they are primarily inflammatory conditions. Similarly, conditions such as acute abscesses are more effectively managed by surgical intervention such as tooth extraction or root canal treatment. ${ }^{1}$ Since the deferral of operative treatment is a risk factor for severe infectious complications from an odontogenic infection, ${ }^{6}$ the management of dental problems in general practice could result in increased patient morbidity. Furthermore, the indiscriminate use of antibiotics may contribute to the emergence of antibiotic resistant bacterial strains.

Despite anecdotal evidence from the general medical profession that attendances for dental problems are increasing, ${ }^{78}$ there has been no prior exploration of the attitudes of GPs towards the management of dental problems in daily practice, and what may influence their decision to prescribe an antibiotic in such consultations. The objectives of this study were therefore to produce an account of the beliefs and attitudes of GPs towards the presentation and management of dental problems in general practice and to explore factors that may influence a practitioner to prescribe antibiotics for patients with tooth-related problems.

\section{METHODS}

\section{Study design}

This was a qualitative, semistructured interview study conducted in Wales, UK, between January and October 2013. This study is reported according to the Consolidated Criteria for Reporting Qualitative Research (COREQ) ${ }^{9}$

\section{Participants and sampling}

The sampling frame comprised all GPs working in Wales. A database of all GPs working in Wales as of October 2012 was compiled from the National Health Service (NHS) Health in Wales Directory. ${ }^{10}$ Since little was known a priori about the attitudes of GPs towards the management of dental problems, maximum variation sampling techniques were employed. This aimed to identify common attitudes that cut across variations such as length of practising career, practice location and patient socioeconomic demographic, while developing multiple perspectives on the topic. Participants were selected on the basis of:

- Practice locality (based on the 2011 Rural-Urban Classification for small area geographies). ${ }^{11}$

- Level of local deprivation (obtained from the Welsh Index of Multiple Deprivation 2011 (WIMD 11), the
Welsh Government's official measure of relative deprivation for small areas in Wales). ${ }^{12}$

- Time since primary medical qualification (based on the General Medical Council online register). ${ }^{13}$

One hundred and seventy GPs were sent a letter of invitation outlining the study. Those who expressed an interest in participating $(n=42)$ were sent further information and a consent form. Of these, 22 provided written consent and 17 were interviewed. Non-responding GPs were not contacted again.

\section{Data collection and analyses}

Semistructured interviews were conducted via telephone by a researcher (AC) trained in qualitative data collection and analysis. All interviews were audio recorded. No notes were made during the interview other than memos of additional questions or probes to ask each participant. This encouraged active listening. Fieldnotes were made at the end of each interview.

An initial interview guide was prepared prior to data collection. This was informed by the scientific literature, topic guides from other studies, and clinical experiences of the research team. However, the schedule was not standardised, nor replicated identically for each interview. The nature of the sample meant some GPs had specific characteristics that required supplementary questioning. Throughout the interviews, questions were added, modified or removed as theories emerged and developed. The interviews were loosely conversational but focused around key predetermined topics, with opportunities for the interviewer to prompt and probe to enable deeper exploration of a concept or idea. No repeat interviews were undertaken.

Interviews were transcribed in full and the interviewer subsequently checked all transcripts with the audio recording to ensure they were a faithful record of the interview, and to aid familiarisation. Transcripts were not returned to participants for comment.

Thematic analysis techniques described by Braun and Clarke $^{14}$ were employed. Analysis was facilitated by the qualitative data analysis software package NVivo. ${ }^{15} \mathrm{~A}$ second researcher experienced in qualitative research (FW) second coded a fifth $(n=3)$ of the transcripts to examine the coding system, and any discrepancies between coders were discussed and resolved. After coding, themes were reviewed, defined and named, before data coded at 'Experience of and attitudes towards dental consultations in general practice' and 'Are antibiotics the answer?' were extracted for further analysis and for purposes of this report.

Data collection, transcription and analysis were undertaken concurrently to examine the emergence of themes and determine the point at which saturation had been reached. Saturation was defined as 'data adequacy', and was reached when the richness of data within a theme no longer appeared to be increasing with subsequent interviews. The interview process ceased when the researcher felt that there was sufficient data to build a 
Table 1 Study participants

\begin{tabular}{llll}
\hline Practitioner ID & Gender & $\begin{array}{l}\text { Number of years } \\
\text { since qualification* }\end{array}$ & Practice descriptort \\
\hline GP1 & Female & $\leq 10$ & Urban \\
GP2 & Female & $>30$ & Rural \\
GP3 & Male & $\leq 10$ & Urban, 20\% most deprived community \\
GP4 & Male & $11-20$ & Rural \\
GP5 & Male & $21-30$ & Urban \\
GP6 & Male & $>30$ & Urban \\
GP7 & Male & $11-20$ & Urban, 20\% least deprived community \\
GP8 & Male & $11-20$ & Urban \\
GP9 & Male & $\leq 10$ & Urban, 20\% most deprived community \\
GP10 & Female & $21-30$ & Urban, 20\% least deprived community \\
GP11 & Female & $21-30$ & Urban \\
GP12 & Female & $11-20$ & Urban, 20\% most deprived community \\
GP13 & Female & $21-30$ & Urban, 20\% most deprived community \\
GP14 & Female & $11-20$ & Urban \\
GP15 & Female & $21-30$ & Urban, 20\% most deprived community \\
GP16 & Male & $21-30$ & Rural \\
GP17 & Male & $21-30$ & Rural \\
\hline
\end{tabular}

${ }^{*}$ Number of years since qualification-since primary medical degree.

†Practice descriptor-determined by 2011 Rural-Urban Classification for small area geographies and WIMD 11 rank, based on practice postcode. ${ }^{11}{ }^{12}$ This table highlights practices in the $20 \%$ most and $20 \%$ least deprived communities in Wales accord to WIMD 11 rank. WIMD, Welsh Index of Multiple Deprivation.

comprehensive and convincing insight into GPs' beliefs and attitudes. This point was judged to have been following coding of the 17 th interview.

\section{Research team and reflexivity}

At the time of the study the interviewer (AC) was a practising dentist undertaking postgraduate research at Cardiff University. Participants were aware of her professional identity prior to the interview. Participants were informed that the study sought to understand how GPs manage patients with dental problems. Immediately prior to the interview it was stressed that the aim of the interview was not to test knowledge but to understand the practitioner's experiences and point of view.

The professional background of the research team, which included an academic GP, a public health dentist and a sociologist helped to ensure that any particular professional biases that may have emerged throughout the analysis were discussed and challenged.

\section{RESULTS}

\section{Sample}

Seventeen GPs participated, of which nine were male (table 1). Interviews lasted on average $23.6 \mathrm{~min}$ (SD $8.0 \mathrm{~min})$.

\section{Experience of and attitudes towards dental consultations} Frequency of dental consultations and perceived reasons for attendance

Among the GPs interviewed reported frequency of dental consultations varied from approximately once a week, to once every few months. While some practitioners reported that the rate of dental consultations had remained relatively stable, others described how the number of patients attending with dental problems had increased or decreased during their time at the practice. Increases were commonly attributed to disruption of local NHS dental services, while decreases were credited to improved access to dentistry, more rigorous practice triaging systems, or the education of patients regarding where to access appropriate dental care.

It may be that they're being given better advice on contacting the practice by telephone and they're being told, 'Look if this is a dental problem you need to see a dentist not a GP and you know you can access a dentist through NHS direct.' It's one of these things that we see I think less frequently than we did a couple of years ago. Whether our behaviour has influenced that by refusing to, well not refusing to treat them, but by being fairly blunt about the fact that they've come to the wrong professional, I don't know, but it certainly feels less of a problem anyway. GP7

Patients attending for dental consultations were typically characterised as likely to be of working-age and socioeconomically deprived. One practitioner described a high prevalence of comorbidities, including mental health problems among patients consulting for toothrelated problems.

They're not so much the younger ones or children, and not so much the older ones. They're mostly, well, twenties to sixties. Bit of wide middle-age range but it's that age range. Possibly skewed a bit to the lower income end. 
Table 2 Reasons described as to why patients with dental problems may consult a GP

\begin{tabular}{|c|c|}
\hline Reason & Example quotation \\
\hline \multicolumn{2}{|l|}{ Access } \\
\hline Shortage of dental services & $\begin{array}{l}\text { "...there's a huge problem with recruitment for dentistry. I would say about five } \\
\text { years ago now, things were desperate then, nobody could get registered." GP2 }\end{array}$ \\
\hline Difficulties accessing dental services & $\begin{array}{l}\text { "The patients often say they can't get in to see the dentist for, you know, a week, a } \\
\text { fortnight, a month or whatever... Or they say that their dentist is twenty miles away } \\
\text { or ten miles away and they can't, they haven't got access to him because they } \\
\text { haven't got transport that day. Whereas the patients with us they tend to be } \\
\text { registered in our locality, so even if they haven't got transport they can still walk to } \\
\text { the surgery." GP6 }\end{array}$ \\
\hline $\begin{array}{l}\text { Comparative ease of accessing } \\
\text { general medical care }\end{array}$ & $\begin{array}{l}\text { "I think a lot of the time these patients don't know how to access the help that they } \\
\text { need and we're very identifiable and very easily accessible." GP3 }\end{array}$ \\
\hline \multicolumn{2}{|l|}{ Practitioner preference } \\
\hline Anxiety & "We do have some patients who say they're too scared to go to the dentist." GP12 \\
\hline $\begin{array}{l}\text { Dissatisfaction with previous dental } \\
\text { treatment }\end{array}$ & $\begin{array}{l}\text { "A lady who'd seen her general dental practitioner the previous day came to me for } \\
\text { a second opinion." GP5 }\end{array}$ \\
\hline \multicolumn{2}{|l|}{ Financial concerns } \\
\hline Cost of prescriptions & $\begin{array}{l}\text { "People are careful how they spend their money and if they can get something free } \\
\text { from the GP, or see a dentist and pay for a prescription..." GP16 }\end{array}$ \\
\hline \multicolumn{2}{|l|}{ Perceived need for antibiotics } \\
\hline Influenced by prior experience & $\begin{array}{l}\text { "Most of the time they have like a preconceived perception of what's going on. So, } \\
\text { most of the time they'll say, 'I think l've got an abscess doc, can you give me some } \\
\text { antibiotics, it worked last time?'" GP9 }\end{array}$ \\
\hline \multicolumn{2}{|l|}{ Referred or poorly differentiated pain } \\
\hline Sinusitis & $\begin{array}{l}\text { "We see patients that might have, or they think they have, sinusitis but it's actually a } \\
\text { dental abscess" GP15 }\end{array}$ \\
\hline Earache & $\begin{array}{l}\text { "We see quite a lot of patients with unexplained earache that they would present to } \\
\text { us, but it's actually toothache." GP15 }\end{array}$ \\
\hline
\end{tabular}

Also skewed in that a significant proportion of them will have other problems particularly mental health issues, and therefore they've not looked after their teeth for many years. GP17

Practitioners described a number of reasons why patients may consult a GP when experiencing dental problem. Of these, difficulties accessing dental services and the comparative ease of access in primary medical care were the most commonly cited explanations (table 2).

\section{Attitudes towards consultations for dental problems}

While there was a consensus among GPs that general practice was not the optimal environment to manage dental problems, a minority of GPs considered dental problems only a marginal inconvenience in their daily practice. These clinicians described how such consultations were typically swift and relatively straight forward, and could even provide an element of relief following more complex patients. These practitioners seemed reconciled to seeing patients with dental problems and preferred to 'grin and bear it' rather than take any action to reduce the frequency of dental consultations.

It is usually a brief consultation which, for me, is quite pleasant to have a short, brief consultation rather than somebody walking in with a list of about ten different things. Yeah? Not that I welcome it, but I don't find it a burden. GP6

However other practitioners expressed feeling exasperation at having to deal with dental consultations, especially when under pressure. This was compounded by the fact they would receive little to no financial remuneration for such consultations.

You know the occasional patient I don't think any of us really mind. They're not long consultations, they're not complex consultations, or challenging consultations, you just get on and do it because it's an emergency consultation. But certainly if you get a week where there are three or four dental patients in and you think, 'That's an hour', when we've got a waiting list. It's a frustration. And then you kind of think, 'This is not something we're going to be directly reimbursed for, either'. GP1

Some GPs were more overtly opposed to seeing patients with dental problems. They perceived dental consultations an abuse of the system which diverted resources away from patients presenting with more appropriate symptoms.

Absolutely a huge time waster... I don't want to give you the impression we're suffering, but I think since the new contract we do thirty percent more work than we safely 
should do, so all it takes is a couple of toothaches on your screen who want a phone call and you're really biting your tongue. GP5

Feelings of animosity towards dental consultations arose primarily due to two reasons. First, practitioners felt they were ill-equipped to treat such problems and consequentially had concerns regarding the possible repercussions of attempting to treat dental problems. Second, GPs felt that their service and accessibility was being taken advantage of.

I have to say rather than seeing it as a burden it would be more that I feel out of my depth. GP12

I think that we are treated as a convenience. GP2

Attitudes towards the presentation and management of dental problems could vary dramatically within a single practice. Some practitioners described colleagues who refused to see patients with dental problems as stubborn or obstinate, although always in good humour. In comparison, practitioners who were strongly opposed to the management of dental conditions in general practice expressed varying degrees of consternation towards colleagues who willingly treated patients presenting with toothache. They described how, in their experience, this led to an increased likelihood of patients consulting during subsequent episodes of dental pain. One GP even explained how differences in opinion regarding the management of dental problems contributed to a breakdown of partner relations within his practice.

However, despite general negativity towards the overall issue of dental problems within their practice, GPs expressed empathy towards patients experiencing dental problems. They appreciated the debilitating effects of dental pain, and were aware of the complexities of accessing emergency dental care. These sentiments illustrated that much of the antagonism GPs expressed in relation to dental consultations were associated with a 'flawed system' that resulted in inadequate access to emergency dental care, rather than directed towards individual patients. The exception to this was patients who were perceived to be attending to avoid costs associated with dental treatment.

The ones that probably annoy us most are the ones that are coming in because they've got a private dentist and they have to pay for their antibiotics. They come into us saying, 'Well I don't want to pay' and you think, 'Well, this is, I suppose, a cost to our service', so they're the ones that annoy, if you like. GP1.

\section{Use of antibiotics in the management of dental problems}

Prescription of antibiotics in the management of dental problems varied dramatically within the sample. Some practitioners reported that they would prescribe an antibiotic to most, if not all patients with a dental problem. In contrast others were much more reluctant to prescribe an antibiotic and would consider prescribing analgesics, or would only provide advice about seeking dental care.

I think generally if it's a dental problem we usually end up prescribing antibiotics. GP1

I'd say the vast majority don't receive anything other than analgesia off me really. GP7

Similarly, there were conflicting opinions among the practitioners as to whether antibiotics were the optimal treatment for acute dental conditions. While some thought that antibiotics were the recommended first-line therapy, others discussed that definitive operative treatment was required. In fact the majority of GPs reported that they had received little or no education at either undergraduate or postgraduate level regarding the diagnosis and management of dental conditions. Therefore, most GPs' dental knowledge had been gained informally from interaction with friends who were dentists, from working alongside dentists in A\&E, or from being a patient themselves. A disadvantage associated with this 'informal' method of learning was the confusion that arose when GPs received mixed messages regarding the management of dental problems, in particular the use of antibiotics.

I've always found it a bit of a dilemma because my own dentist, I've had two since I've been in [NAME PLACE], one of whom said, 'I wish GPs wouldn't give antibiotics because often the patient won't consult until several days later and it's harder for me to unravel what's gone on before it', and the other one who said he thought GPs were very mean if they didn't treat someone who's in pain and needed antibiotics to tide them over. GP12

When managing patients with dental problems GPs described how they balanced trying to provide symptomatic relief against trying to motivate patients to access more appropriate sources of care for tooth-related pain. GPs who reported that they were more likely to prescribe an antibiotic generally did so because they prioritised a patient's immediate needs and wished to minimise the likelihood of clinical deterioration.

I'm perhaps a bit soft and, at the end of the day if your suspicions are that, you know, there is a dental abscess or there is an infection there I don't believe in saying, 'Oh well, you know, you're going to see a dentist tomorrow, wait 'til then'. My feeling is, you know, most of the time when you're seeing it, it is genuine and, you know, I'm quite convinced that there is an infection there so I'll crack on and give antibiotics. GP9

However, there was recognition within this group of practitioners that this may encourage reattendance during subsequent episodes of dental pain. In a similar way, practitioners who reported rarely prescribing antibiotics for dental conditions often did so to motivate 
patients to seek more appropriate care which they believed would lead to quicker resolution of pain.

I try very hard not to [prescribe antibiotics] because then I feel it will close the loop and they will then have the expectation that they don't actually need dental care. GP13

Several GPs described how reduction of emergency dental services during weekends or holiday periods would increase their likelihood of prescribing an antibiotic. Similarly, one practitioner described how their rate of antibiotic prescription for dental problems had decreased since improvements in access to local emergency dental services. This, they explained, meant that they were able to direct patients to a source of more appropriate care and felt less obliged to try and manage the condition themselves.

\section{Patient expectations and requests for antibiotics}

GPs perceived high levels of expectation for antibiotics among patients consulting for dental conditions and reported that many patients often requested antibiotics when consulting for tooth-related problems.

Ninety percent of the patients that I have seen are more or less convinced that it's a dental abscess and they need antibiotics. They are aware of it and they come asking for it. GP4

Many practitioners, particularly those who did not routinely prescribe antimicrobials for dental problems, were prepared to decline requests for antibiotics, even if it led to patient dissatisfaction.

INT-How do patients react when you tell them that you're not going to give them antibiotics?

GP -Some of them are upset and will go and ask the receptionist if they can see the other doctor instead. GP17

However, a minority of practitioners reported that they would be more likely to prescribe an antibiotic to dental patients who asked for one. These practitioners were likely to have a moderate attitude towards dental consultations in general practice and prescribed antibiotics in these situations to appease the patient and avoid conflict.

Well there's certainly patient pressure there to prescribe. Very often, to be quite honest with you, it's easier just to prescribe than spend time arguing. GP2.

\section{DISCUSSION}

This study aimed to explore GPs' views regarding the presentation and management of dental problems in general practice and, to our knowledge, is the first to describe factors that may influence their use of antibiotics in such consultations. Attitudes to dental consultations varied, with some clinicians viewing dental consultations as a minor frustration, while others expressed more serious grievances. Practitioners' attitudes were principally influenced by the perceived burden of dental conditions, general pressures of their workload and perceptions about why the patient had been motivated to seek care. While there was a consensus that general practice was not the best setting for managing dental problems, GPs expressed sympathy towards patients experiencing toothache who had been driven to consult after being unable to access emergency dental care. Although most practitioners admitted they had received little or no formal teaching in the management of dental problems, many reported routinely prescribing antibiotics for tooth-related conditions. The decision to prescribe antibiotics depended on clinicians' awareness regarding the appropriate use of antibiotics in the management of dental conditions, and how they balanced the immediate needs of their patients against a desire to motivate patients to seek more appropriate care. Patients consulting with a dental problem were perceived as frequently expecting to receive an antibiotic, and a minority of GPs described that this may make them more likely to prescribe.

The reported frequency of dental consultations varied between practitioners; while some saw patients with tooth-related problems on a weekly basis, others reported that dental consultations were a much rarer occurrence in their practice. This is broadly consistent with a previous study which reported that rates of attendance for dental consultations varied substantially between practices in Wales. ${ }^{2}$ However, despite concerns within the general medical profession, ${ }^{78}$ there has been few attempts to describe current rates of attendance for tooth-related conditions in general practice in the UK within the past decade. During this time there has also been the introduction of a new NHS dental contract between dentists and commissioning bodies in England and Wales. This contract, launched in April 2006, resulted in changes to the provision of, and access to, primary dental care services and means patients were no longer 'registered' with a dental practitioner unless undergoing active treatment. Since access to dentistry may be a key driver of dental consultations in general medical practice, it is likely that rates of consultations for dental conditions may have changed from those reported in previous studies. ${ }^{2}$ Further work is therefore required to quantify the burden of dental consultations on general medical services in the UK.

This study sought to understand why patients with dental problems may consult their GP. Previous patient surveys have indicated that choice of primary care practitioner during episodes of dental problems may be influenced by: access to healthcare services; dental anxiety; costs associated with treatment; presentation of dentoalveolar pain, and patients' preferences regarding healthcare practitioners. ${ }^{16-19}$ All of these factors were 
discussed to a greater or lesser extent by the GPs interviewed, with difficulties accessing dental care being the most commonly cited explanation. However, it is unclear whether patients are motivated by the reasons GPs think they are. Patients may be unwilling to disclose to their doctor the reasons why they are attending, especially if it is related to financial or other socially-sensitive reasons. Alternatively, GPs may not routinely enquire as to why patients did not seek care from a dental practitioner and therefore answers may be based on their own preconceptions. Consequently more patient-centred research is required to investigate the motivations underlying consultations for dental problems in general practice.

Attitudes towards the presentation and management of patients with dental problems varied not only between GPs, but according to the specific situation in which the consultation occurred. Practitioners' feelings were related to the burden of seeing dental conditions on their day-to-day practice, perceptions about why the patient was seeking care for their dental problem and beliefs about the appropriateness of such consultations. While these findings are broadly supported by anecdotal evidence, ${ }^{78}$ results may not be generalisable to a wider population of GPs. Furthermore, clinicians who agreed to take part may have had a special interest in this topic, and therefore it is possible that we missed important data obtainable only from GPs who did not wish to participate.

Most of the GPs interviewed had a limited understanding of the aetiology and management of dental problems. This finding is supported by previous studies which have described poor diagnostic awareness of orofacial conditions and dental emergencies among physicians. $^{20-22}$ The most likely reason for this is lack of training, with only $52 \%$ of UK undergraduate medical curricula including teaching about oral pathologies. ${ }^{21}$ Lack of instruction in the optimal management of acute dental conditions may be one of the principal reasons why antibiotics are so frequently prescribed in the management of dental problems in general practice. However, while raising awareness of the importance of operative treatment in the management of dental problems among GPs may result in fewer patients receiving antibiotics, general practice remains an inappropriate environment for the management of dental problems. Therefore, more needs to be done to assist patients in accessing appropriate dental care when they experience a tooth-related problem. This may be achieved through better communication about the availability of local dental services by dental providers, or campaigns to inform patients about the appropriate healthcare professional to consult when experiencing dental problems.

\section{CONCLUSIONS}

Dental consultations were seen to make up a regular part of many GPs' workload, although opinions varied as to the burden this presented. While the GPs within this study recognised that dental problems were not optimally managed in general practice, many participants believed that they were meeting a need that resulted from what they saw as a 'flawed' dental service. Practitioners had generally received little training related to oral health, and patients seeking care for dental problems often only received prescriptions for antibiotics or analgesics. Attitudes towards the prescription of antibiotics varied dramatically within the sample, with some clinicians prescribing during the majority of consultations for dental conditions, while others were more reluctant to prescribe such agents. As such, patients are likely to receive different messages about the appropriateness of seeking care for dental problems in general practice, which may affect their future consultation behaviour.

The prescription of antibiotics by GPs in the management of dental problems may contribute to both patient morbidity from untreated dental disease and the emergence of antimicrobial resistance. Interventions are therefore needed to support patients in accessing appropriate care when experiencing dental conditions. This research has also identified the need for quantitative data on general practice consultations for dental problems and qualitative research exploring patient perspectives on reasons for consulting.

Acknowledgements The authors would like to thank the GPs who participated in this study, and colleagues in the School of Medicine who assisted in the transcription of interviews.

Contributors ALC contributed to the study design, developed the interview guide, conducted the interviews, analysed results and wrote the initial draft of the manuscript. FW contributed to the study design, developed the interview guide, analysed results and revised the manuscript. NAF and IGC contributed to the study design and revision of the manuscript.

Funding The work was supported by: the Wales School for Primary Care Research (grant number 504746); a President's Research Scholarship from Cardiff University (grant number RCUC099).

Competing interests None declared.

Ethics approval The study was reviewed and given a favourable ethical opinion by the London Central Proportionate Review Committee (ref: 12/LO/ 1213).

Provenance and peer review Not commissioned; externally peer reviewed.

Data sharing statement No additional data are available.

Open Access This is an Open Access article distributed in accordance with the Creative Commons Attribution Non Commercial (CC BY-NC 4.0) license, which permits others to distribute, remix, adapt, build upon this work noncommercially, and license their derivative works on different terms, provided the original work is properly cited and the use is non-commercial. See: http:// creativecommons.org/licenses/by-nc/4.0/

\section{REFERENCES}

1. Scottish Dental Clinical Effectiveness Programme. Drug prescribing for dentistry: dental clinical guidance. 2nd edn. Dundee: Scottish Dental Clinical Effectiveness Programme, 2011.

2. Anderson R, Richmond S, Thomas DW. Patient presentation at medical practices with dental problems: an analysis of the 1996 General Practice Morbidity Database for Wales. Br Dent J 1999;186:297-300. 
3. Ashworth $\mathrm{M}$, Charlton J, Ballard $\mathrm{K}$, et al. Variations in antibiotic prescribing and consultation rates for acute respiratory infection in UK general practices 1995-2000. Br J Gen Pract 2005;55: 603-8.

4. Currie CJ, Berni E, Jenkins-Jones S, et al. Antibiotic treatment failure in four common infections in UK primary care 1991-2012 longitudinal analysis. BMJ 2014:349:g5493.

5. Anderson R, Calder L, Thomas DW. Antibiotic prescribing for dental conditions: general medical practitioners and dentists compared. Br Dent J 2000;188:398-400.

6. Seppänen L, Lemberg KK, Lauhio A, et al. Is dental treatment of an infected tooth a risk factor for locally invasive spread of infection? J Oral Maxillofac Surg 2011;69:986-93.

7. Bint A. NHS dentistry: general practitioners are doing dentists' work. BMJ 2008;336:1088.

8. Matthews-King A. Nine out of ten GPs feel 'under pressure' from patients with dental complaints. 2013. http://www.pulsetoday.co.uk/ news/clinical-news/nine-out-of-ten-gps-feel-under-pressure-frompatients-with-dental-complaints/20005094.article\#.VEo8t_14rYg (accessed 24 Oct 2014).

9. Tong A, Sainsbury P, Craig J. Consolidated criteria for reporting qualitative research (COREQ): a 32-item checklist for interviews and focus groups. Int J Qual Health Care 2007:19:349-57.

10. NHS Wales. Health in Wales Directory. 2012. http://www.wales.nhs. uk/ourservices/directory (accessed 27 Nov 2012).

11. Office for National Statistics. 2011 Rural-Urban Classification for small area geographies. 2013. http://www.ons.gov.uk/ons/ guide-method/geography/products/area-classifications/ 2011-rural-urban/index.html (accessed 27 Nov 2014).
12. Statistics for Wales. Welsh Index of Multiple Deprivation (WIMD). 2011. http://wales.gov.uk/statistics-and-research/ welsh-index-multiple-deprivation/?lang=en\#/statistics-and-research/ welsh-index-multiple-deprivation/?lang=en (accessed 24 Nov 2014).

13. General Medical Council. List of registered medical practitioners. 2010. http://www.gmc-uk.org/ (accessed 1 July 2013).

14. Braun V, Clarke V. Using thematic analysis in psychology. Qual Res Psychol 2006;3:77-101.

15. QSR International Pty Ltd. NVivo qualitative data analysis software. Version 8. Victoria: QSR International Pty Ltd; 2008.

16. Freeman $R$. The psychology of dental patient care: barriers to accessing dental care: patient factor. Br Dent $J$ 1999;187:141-4.

17. Nuttal N, Freeman R, Bevean-Seymour C, et al. 8: Access and barriers to care-a report from the Adult Dental Health Survey. 2009. Leeds: The Health and Social Care Information Centre, 2011.

18. Mansour MH, Cox SC. Patients presenting to the general practitioner with pain of dental origin. Med J Aust 2006;185:64-7.

19. Bell GW, Smith GL, Rodgers JM, et al. Patient choice of primary care practitioner for orofacial symptoms. Br Dent $J$ 2008;204:669-73.

20. Trivedy C, Kodate N, Ross A, et al. The attitudes and awareness of emergency department (ED) physicians towards the management of common dentofacial emergencies. Dent Traumatol 2012;28:121-6.

21. McCann PJ, Sweeney MP, Gibson J, et al. Training in oral disease, diagnosis and treatment for medical students and doctors in the United Kingdom. Br J Oral Maxillofac Surg 2005;43:61-4.

22. Bissett SM, Stone KM, Rapley T, et al. An exploratory qualitative interview study about collaboration between medicine and dentistry in relation to diabetes management. BMJ Open 2013;3:pii: e002192. 